$\underline{\mathbf{P}-14}$

\title{
Influence of Zerumbone Supplementation a Natural Dietary Product from Zingiber Zerumbet Smith on Early-Developed Atherosclerotic Lesions in Cholesterol-Fed Rabbits
}

\author{
Hemen, H.O ${ }^{1, *}$, Rasedee Abdullah ${ }^{1}$, Hazilawati, $\mathrm{H}^{1}$, Heshu, S.R., Noordin, M.M ${ }^{1,{ }^{*}}$ and Zuki, A.B.Z ${ }^{2}$ \\ ${ }^{I}$ Department of Pathology and Microbiology, ${ }^{2}$ Department of Preclinical Sciences, Faculty of veterinary Medicine, \\ Universiti Putra Malaysia, 43400 UPM, Serdang, Malaysia; E-mail: noordin@vet.upm.edu.myhimenho75@yahoo.com
}

\begin{abstract}
Atherosclerosis is a condition of the arterial wall to 'injury', which is prominently driven by inflammatory responses. The objective of this investigation is to evaluate the preventive and therapeutic efficacy of zerumbone (ZER), a natural cyclic sesquiterpene isolated from Zingiber zerumbet Smith against early-developed atherosclerotic lesion in the aorta of New Zealand white rabbits fed a cholesterol-rich diet. Forty two male rabbits used in the present study were randomly assigned into seven experimental groups as follows: EG-I considered as control group was fed a standard rabbit pellet diet, EG-II given a cholesterol-rich diet (1\%), EG-III given ZER $(0.4 \%)$ two weeks before day 0 as preventive measure and thereafter with the course of cholesterol-rich diet, EG-IV given ZER (0.8\%), EG-V given ZER (1\%), EG-VI given Simvastatin (SIM) (20 mg/Kg) and EG-VII given ZER $(0.8 \%)$ together with SIM $(20 \mathrm{mg} / \mathrm{Kg})$. Experimental groups from EG-IV to VII acted to represent the therapeutic measure of ZER against early-developed atherosclerosis. Tissue samples were collected from the thoracic aorta and aortic arch at 12 weeks post-feeding for gross inspection, histopathological and ultrastructural (scanning electron microscopy) examination. Morphological analysis revealed that endothelial damage and atheromas plague built up were notably diminished in ZER treated groups in a dose dependent manner where it is more prominent in EG-III and EG-VII compared to EGs-VI, V, and VI. However, most of the ZER treated groups showed obvious reduction in plague development in contrast to cholesterolrich diet group EG-II. Our data indicate that dietary intake of ZER considerably averts and decreases early plague formation and development via significant lessening in smooth muscle cells proliferation and reduction of inflammatory progression beside its hypolipidemic effect (Data not shown).
\end{abstract}

Keywords: Rabbits, Atherosclerosis, Zerumbone, Histopathology, Ultramorphology. 